\title{
Dynamics of a Discrete Allelopathic Phytoplankton Model with Infinite Delays and Feedback Controls
}

\author{
Liang Zhao (D), ${ }^{1,2}$ Bin Qin, ${ }^{2}$ and Fengde Chen ${ }^{3}$ \\ ${ }^{1}$ School of Mathematics and Informatics, Fujian Normal University, Fuzhou, Fujian 350117, China \\ ${ }^{2}$ College of Information and Statistics, Guangxi University of Finance and Economics, Nanning, Guangxi 530003, China \\ ${ }^{3}$ College of Mathematics and Computer Science, Fuzhou University, Fuzhou, Fujian 350116, China
}

Correspondence should be addressed to Liang Zhao; zhaoliang200809@yeah.net

Received 30 July 2019; Revised 6 December 2019; Accepted 19 December 2019; Published 13 January 2020

Academic Editor: Leonid Shaikhet

Copyright (c) 2020 Liang Zhao et al. This is an open access article distributed under the Creative Commons Attribution License, which permits unrestricted use, distribution, and reproduction in any medium, provided the original work is properly cited.

\begin{abstract}
A discrete allelopathic phytoplankton model with infinite delays and feedback controls is studied in this paper. By applying the discrete comparison theorem, a set of sufficient conditions which guarantees the permanence of the system is obtained. Also, by constructing some suitable discrete Lyapunov functionals, some sufficient conditions for the extinction of the system are obtained. Our results extend and supplement some known results and show that the feedback controls and toxic substances play a crucial role on the permanence and extinction of the system.
\end{abstract}

\section{Introduction}

Given a bounded sequence of real numbers $f(k)$, let $f^{u}$ and $f^{l}$ denote $\sup _{k \in Z} f(k)$ and $\inf _{k \in Z} f(k)$, respectively.

Many real-world phenomena are studied through discrete mathematical models governed by difference equations which are more appropriate than the continuous ones when the populations have nonoverlapping generations; the study of the dynamic behaviors of discrete time models becomes the subject of intense research in mathematics biology, such topics as permanence and extinction, and existence of positive periodic solution (almost periodic solution), have been extensively studied by many scholars (see [1-23] and the references cited therein).

Recently, some scholars believed that a more appropriate competition model should be considered with nonlinear interinhibition terms. Qin et al. [1] and Wang et al. [2] considered the following discrete two species competitive system with nonlinear interinhibition terms:

$$
\begin{aligned}
& x_{1}(k+1)=x_{1}(k) \exp \left\{r_{1}(k)-a_{1}(k) x_{1}(k)-\frac{c_{2}(k) x_{2}(k)}{1+x_{2}(k)}\right\}, \\
& x_{2}(k+1)=x_{2}(k) \exp \left\{r_{2}(k)-a_{2}(k) x_{2}(k)-\frac{c_{1}(k) x_{1}(k)}{1+x_{1}(k)}\right\},
\end{aligned}
$$

where $r_{i}(k), a_{i}(k), c_{i}(k)(i=1,2)$ are assumed to be bounded positive sequences, and $x_{1}(k)$ and $x_{2}(k)$ represent the density of species $x_{1}$ and $x_{2}$ at the $k$ th generation, respectively, $r_{i}(k)(i=1,2)$ is the intrinsic growth rate of two species. $a_{i}(k)(i=1,2)$ is the rate of intraspecific competition of the first and second species, respectively. $c_{i}(k),(i=$ $1,2)$ is the rate of interspecific competition of the first and second species, respectively. Qin et al. [1] obtained sufficient conditions which ensure the permanence, existence, and global stability of positive periodic solutions of system (1). As for the almost periodic case, Wang and Liu [2] further 
investigated the existence, uniqueness, and uniformly asymptotic stability of positive almost periodic solution of system (1).

In recent years, many scholars have made great achievements in the competition system with the effect of toxic substance [3, 4, 24-26]. Yue [3] investigated system (1) with the second species which could be toxic, while the other one is nontoxic. He studied system (2) and gave the sufficient conditions of the extinction of one species and the global attractive of the other one.

$$
\begin{aligned}
& x_{1}(k+1)=x_{1}(k) \exp \left\{r_{1}(k)-a_{1}(k) x_{1}(k)-\frac{c_{2}(k) x_{2}(k)}{1+x_{2}(k)}-b_{1}(k) x_{1}(k) x_{2}(k)\right\}, \\
& x_{2}(k+1)=x_{2}(k) \exp \left\{r_{2}(k)-a_{2}(k) x_{2}(k)-\frac{c_{1}(k) x_{1}(k)}{1+x_{1}(k)}\right\} .
\end{aligned}
$$

Note that ecosystems are easily disturbed by human activities, such as planting and harvesting, which can give rise to changes of population density. In order to give a better description of such a system, scholars introduced feedback control variables into ecosystems. Many researchers have done research on the systems with feedback control variables $[6-8,11,14,16,17]$. Recently, Wang and Liu [6] studied the competitive system with feedback controls as follows:

$$
\begin{aligned}
& x_{1}(n+1)=x_{1}(n) \exp \left\{r_{1}(n)-a_{1}(n) x_{1}(n)-\frac{b_{1}(n) x_{2}(n)}{1+x_{2}(n)}-e_{1}(n) u_{1}(n)\right\}, \\
& x_{2}(n+1)=x_{2}(n) \exp \left\{r_{2}(n)-\frac{b_{2}(n) x_{1}(n)}{1+x_{1}(n)}-a_{2}(n) x_{2}(n)-e_{2}(n) u_{2}(n)\right\}, \\
& \Delta u_{1}(n)=-b_{1}(n) u_{1}(n)+d_{1}(n) x_{1}(n), \\
& \Delta u_{2}(n)=-b_{2}(n) u_{2}(n)+d_{2}(n) x_{2}(n),
\end{aligned}
$$

where $u_{i}(n), i=1,2$, denotes the feedback control variables. Wang and Liu [6] studied the existence and uniformly asymptotic stability of unique positive almost periodic solution of system (3). Yu [7] further investigated the influence of feedback control variables on the extinction of the system. He gave a set of sufficient conditions for the extinction of one species.
As we all know, due to seasonal fluctuations in the environment and hereditary factors, time delays have been introduced into the biological system (see [11, 14, 21, 27-36]). Zhao et al. [8] further considered a discrete Lotka-Volterra competition system with infinite delays and single feedback control variable as follows:

$$
\begin{aligned}
& x_{1}(n+1)=x_{1}(n) \exp \left\{a_{1}(n)-b_{11}(n) x_{1}(n)-b_{12}(n) \sum_{s=0}^{\infty} k_{12}(s) x_{2}(n-s)-d_{1}(n) \sum_{s=0}^{\infty} h_{1}(s) u(n-s)\right\} \\
& x_{2}(n+1)=x_{2}(n) \exp \left\{a_{2}(n)-b_{22}(n) x_{2}(n)-b_{21}(n) \sum_{s=0}^{\infty} k_{21}(s) x_{1}(n-s)-d_{2}(n) \sum_{s=0}^{\infty} h_{2}(s) u(n-s)\right\} \\
& u(n+1)=u(n)(1-e(n))+c_{1}(n) \sum_{s=0}^{\infty} l_{1}(s) x_{1}(n-s)+c_{2}(n) \sum_{s=0}^{\infty} l_{2}(s) x_{2}(n-s) .
\end{aligned}
$$

By applying the discrete comparison theorem, a set of sufficient conditions which guarantees the permanence of the system is obtained. Also, by constructing some suitable discrete Lyapunov functionals, some sufficient conditions for the global attractivity and extinction of the system are obtained.

Through the above discussion, we find that, based on system (2), we can further consider the infinite delay and 
feedback control. So, we propose and study the following discrete competition system with infinite delays and feedback control variables:

$$
\begin{aligned}
& x_{1}(k+1)=x_{1}(k) \exp \left\{r_{1}(k)-a_{1}(k) x_{1}(k)-\frac{b_{1}(k) \sum_{s=0}^{\infty} K_{12}(s) x_{2}(k-s)}{1+\sum_{s=0}^{\infty} K_{12}(s) x_{2}(k-s)}-\gamma(k) x_{1}(k) x_{2}^{2}(k)-c_{1}(k) \sum_{s=0}^{\infty} H_{1}(s) u_{1}(k-s)\right\}, \\
& x_{2}(k+1)=x_{2}(k) \exp \left\{r_{2}(k)-\frac{b_{2}(k) \sum_{s=0}^{\infty} K_{21}(s) x_{1}(k-s)}{1+\sum_{s=0}^{\infty} K_{21}(s) x_{1}(k-s)}-a_{2}(k) x_{2}(k)-c_{2}(k) \sum_{s=0}^{\infty} H_{2}(s) u_{2}(k-s)\right\}, \\
& u_{1}(k+1)=u_{1}(k)\left(1-e_{1}(k)\right)+d_{1}(k) \sum_{s=0}^{\infty} L_{1}(s) x_{1}(k-s), \\
& u_{2}(k+1)=u_{2}(k)\left(1-e_{2}(k)\right)+d_{2}(k) \sum_{s=0}^{\infty} L_{2}(s) x_{2}(k-s) .
\end{aligned}
$$

In system (4), $x_{i}(k)(i=1,2)$ is the density of $x_{i}$ species at the $k$ th generation, and $u_{i}(k), i=1,2$, is the feedback control variable.

Throughout this paper, we assume that

$\left(\mathbf{C}_{1}\right) r_{i}(k), a_{i}(k), b_{i}(k), c_{i}(k), d_{i}(k)$, and $e_{i}(k)(i=1,2)$ are bounded sequences of real numbers defined on $Z$ such that

$$
\begin{aligned}
& r_{i}^{l}>0, \\
& a_{i}^{l}>0, \\
& b_{i}^{l}>0, \\
& c_{i}^{l}>0, \\
& d_{i}^{l}>0, \\
& 0<e_{i}^{l}<e_{i}^{u}<1, \quad i=1,2 .
\end{aligned}
$$

$\left(\mathbf{C}_{2}\right) K_{i j}(s), H_{i}(s)$, and $L_{i}(s)(i, j=1,2)$ are nonnegative bounded sequences such that

$$
\begin{aligned}
\sum_{s=0}^{\infty} K_{i j}(s) & =1, \\
\sum_{s=0}^{\infty} H_{i}(s) & =1, \\
\sum_{s=0}^{\infty} L_{i}(s) & =1, \quad i, j=1,2, \\
\Theta_{i j} & =\sum_{s=0}^{\infty} K_{i j}(s), \quad s<+\infty, \\
\Lambda_{i} & =\sum_{s=0}^{\infty} H_{i}(s), \quad s<+\infty, \\
\Upsilon_{i} & =\sum_{s=0}^{\infty} L_{i}(s), \quad s<+\infty .
\end{aligned}
$$

According to the biological background of system (5), we only consider the solution of system (5) with the following initial conditions:

$$
\begin{aligned}
& x_{i}(s)=\Psi_{i}(s) \geq 0, \\
& \Psi_{i}(0)>0, \\
& \sup _{k \in Z^{-}} \Psi_{i}(k)<+\infty, \\
& u_{i}(s)=\Phi(s) \geq 0, \\
& \Phi_{i}(0)>0, \\
& \sup _{k \in Z^{-}} \Phi_{i}(k)<+\infty, \\
& \quad i=1,2,
\end{aligned}
$$

where $s=\ldots,-n,-n+1, \ldots,-1,0$. It is easy to prove that the solution of system (5) which satisfies the initial conditions (8) is positive.

Here, we mention that this is the first time such kind of model be proposed and studied, and as far as system (5) is concerned, whether the feedback control variables and toxic substances have influence on the permanence and extinction of the system or not is an interesting problem. The aim of this paper is to investigate the permanence and extinction of system (5); specially, we will find out the answer to the above problem.

The paper is structured in the following way. Some useful Lemmas are presented in Section 2. In Sections 3 and 4, by using the methods of Zhao et al. [8], we investigate the permanence and extinction of system (5). Three examples are presented to show the feasibility of the main results in Section 5 . We end this paper by a brief discussion.

\section{Lemmas}

Now, let us consider the following difference equation:

$$
y(k+1)=a y(k)+b,
$$


where $a$ and $b$ are positive constants.

Lemma 1 (see [37]). Assume that $|a|<1$, for any initial value $y(0)$, there exists a unique solution $y(k)$ of equation (9), which can be expressed as follows:

$$
y(k)=a^{k}\left(y(0)-y^{*}\right)+y^{*}
$$

where $y^{*}=b /(1-a)$. Thus, for any solution $y(k)$ of system (9), we have

$$
\lim _{k \longrightarrow+\infty} y(k)=y^{*}
$$

Lemma 2 (see [37]). Let $k \in N_{k_{0}}^{+}=\left\{k_{0}, k_{0}+1, \ldots, k_{0}+l\right.$ $, \ldots\}, r \geq 0$. For any fixed $k, g(k, r)$ is a nondecreasing function with respect to $r$, and for $k \geq k_{0}$, the following inequalities hold:

$$
\begin{aligned}
& y(k+1) \leq g(k, y(k)), \\
& u(k+1) \geq g(k, u(k)) .
\end{aligned}
$$

If $y\left(k_{0}\right) \leq u\left(k_{0}\right)$, then $y(k) \leq u(k)$ for all $k \geq k_{0}$.

Lemma 3 (see [38]). Assume that $r(n)>0, x(n)$ satisfies $x(n)>0$ and

$$
x(n+1) \leq x(n) \exp \{r(n)(1-a x(n))\},
$$

for $n \in\left[n_{1}, \infty\right)$, where $a$ is a positive constant. Then,

$$
\limsup _{n \longrightarrow+\infty} x(n) \leq \frac{1}{a r^{u}} \exp \left(r^{u}-1\right) \text {. }
$$

Lemma 4 (see [38]). Assume that $r(n)>0, x(n)$ satisfies $x(n)>0$ and

$$
x(n+1) \geq x(n) \exp \{r(n)(1-a x(n))\},
$$

for $n \in\left[n_{1}, \infty\right)$, lim sup $\sin _{n} x(n) \leq x^{*}$ and $x\left(n_{1}\right)>0$, where $a$ and $x^{*}$ are positive constants such that $a x^{*}>1$. Then,

$$
\liminf _{n \longrightarrow+\infty} x(n) \geq \frac{1}{a} \exp \left(r^{u}\left(1-a x^{*}\right)\right) .
$$

Lemma 5 (see [38]). Let $x: Z \longrightarrow R$ be a nonnegative bounded sequence, and let $H: Z_{+} \longrightarrow R$ be a nonnegative sequence such that $\sum_{n=0}^{\infty} H(n)=1$, then

$$
\begin{aligned}
\liminf _{n \longrightarrow+\infty} x(n) & \leq \liminf _{n \longrightarrow+\infty} \sum_{s=-\infty}^{n} H(n-s) x(s) \\
& \leq \limsup _{n \longrightarrow+\infty} \sum_{s=-\infty}^{n} H(n-s) x(s) \leq \limsup _{n \longrightarrow+\infty} x(n) .
\end{aligned}
$$

\section{Permanence}

Concerned with the permanence of system (5), we have the following result.

Theorem 1. Assume that

$$
\begin{array}{r}
r_{i}^{l}-b_{i}^{u} M_{j}-c_{i}^{u} U_{i}>0, \quad i, j=1,2, i \neq j, \\
\gamma^{u}<\frac{r_{1}^{l}-b_{1}^{u} M_{2}-c_{1}^{u} U_{1}}{M_{1} M_{2}^{2}},
\end{array}
$$

hold; then, for any positive solution $\left(x_{1}(k), x_{2}(k), u_{1}\right.$ $\left.(k), u_{2}(k)\right)$ of system (5), we have

$$
\begin{aligned}
& m_{i} \leq \liminf _{k \longrightarrow+\infty} x_{i}(k)<\limsup _{k \longrightarrow+\infty} x_{i}(k) \leq M_{i}, \\
& B_{i} \leq \liminf _{k \longrightarrow+\infty} u_{i}(k)<\limsup _{k \longrightarrow+\infty} u_{i}(k) \leq U_{i},
\end{aligned}
$$

where

$$
\begin{aligned}
M_{i}= & \frac{1}{a_{i}^{l}} \exp \left(r_{i}^{u}-1\right), \\
U_{i}= & \frac{d_{i}^{u} M_{i}}{e_{i}^{l}}, \\
m_{1}= & \frac{r_{1}^{l}-b_{1}^{u} M_{2}-\gamma^{u} M_{1} M_{2}^{2}-c_{1}^{u} U_{1}}{a_{1}^{u}} \\
& \cdot \exp \left(r_{1}^{l}-b_{1}^{u} M_{2}-\gamma^{u} M_{1} M_{2}^{2}-c_{1}^{u} U_{1}-a_{1}^{u} M_{1}\right), \\
m_{2}= & \frac{r_{2}^{l}-b_{2}^{u} M_{1}-c_{2}^{u} U_{2}}{a_{2}^{u}} \cdot \exp \left(r_{2}^{l}-b_{2}^{u} M_{1}-c_{2}^{u} U_{2}-a_{2}^{u} M_{2}\right), \\
B_{i}= & \frac{d_{i}^{l} m_{i}}{e_{i}^{u}}, \quad i=1,2 .
\end{aligned}
$$

Proof. From the first and second equations of system (5), we have

$$
x_{i}(k+1) \leq x_{i}(k) \exp \left[r_{i}(k)\left(1-\frac{a_{i}^{l}}{r_{i}^{u}} x_{i}(k)\right)\right], \quad i=1,2 .
$$

Hence, from Lemma 3, we can obtain

$$
\limsup _{k \longrightarrow+\infty} x_{i}(k) \leq \frac{1}{a_{i}^{l}} \exp \left(r_{i}^{u}-1\right) \stackrel{\text { def }}{=} M_{i} .
$$

According to Lemma 5 and the above inequality, we have

$$
\begin{aligned}
\limsup _{k \longrightarrow+\infty} \sum_{s=0}^{\infty} L_{i}(s) x_{i}(k-s) & =\limsup _{k \longrightarrow+\infty} \sum_{s=0}^{\infty} L_{i}(k-s) x_{i}(s) \\
& \leq \limsup _{k \longrightarrow+\infty} x_{i}(k) \leq M_{i} .
\end{aligned}
$$

For any $\varepsilon>0$, there exists a positive integer $N_{1}$ such that

$$
\sum_{s=0}^{\infty} L_{i}(s) x_{i}(k-s) \leq M_{i}+\varepsilon, \quad \text { for all } n>N_{1} .
$$

By the third and fourth equations of system (5), we have 


$$
u_{i}(k+1) \leq\left(1-e_{i}^{l}\right) u_{i}(k)+d_{i}^{u}\left(M_{i}+\varepsilon\right) .
$$

Hence, by applying Lemmas 1 and 2 to (25), we obtain

$$
\limsup _{k \longrightarrow+\infty} u_{i}(k) \leq \frac{d_{i}^{u}\left(M_{i}+\varepsilon\right)}{e_{i}^{l}} .
$$

Setting $\varepsilon \longrightarrow 0$, it follows that

$$
\limsup _{k \longrightarrow+\infty} u_{i}(k) \leq \frac{d_{i}^{u} M_{i}}{e_{i}^{l}} \stackrel{\text { def }}{=} U_{i} .
$$

Condition (18) implies that, for enough small positive constant $\varepsilon_{1}$, the following inequalities hold:

$$
\begin{array}{r}
r_{1}^{l}-b_{1}^{u}\left(M_{2}+\varepsilon_{1}\right)-\gamma^{u}\left(M_{1}+\varepsilon_{1}\right)\left(M_{2}+\varepsilon_{1}\right)^{2}-c_{1}^{u}\left(U_{1}+\varepsilon_{1}\right)>0, \\
r_{2}^{l}-b_{2}^{u}\left(M_{1}+\varepsilon_{1}\right)-c_{2}^{u}\left(U_{2}+\varepsilon_{1}\right)>0 .
\end{array}
$$

For above $\varepsilon_{1}$, it follows from (22) and (27) that there exists a positive integer $N_{2} \geq N_{1}$ such that

$$
\begin{aligned}
& \sum_{s=0}^{\infty} H_{i}(s) u_{i}(k-s) \leq U_{i}+\varepsilon_{1}, \\
& \sum_{s=0}^{\infty} K_{j i}(s) x_{i}(k-s) \leq M_{i}+\varepsilon_{1}, \\
& x_{i}(k) \leq M_{i}+\varepsilon_{1} \text { for all } n>N_{2} .
\end{aligned}
$$

Thus, for all $n>N_{2}$, from (28), (29), and the first two equations of system (5), we have

$$
\begin{aligned}
& x_{1}(k+1) \geq x_{1}(k) \exp \left\{\left(r_{1}^{l}-b_{1}^{u}\left(M_{2}+\varepsilon_{1}\right)-\gamma^{u}\left(M_{1}+\varepsilon_{1}\right)\right.\right. \\
&\left.\left.\cdot\left(M_{2}+\varepsilon_{1}\right)^{2}-c_{1}^{u}\left(U_{1}+\varepsilon_{1}\right)\right)\left(1-D_{1}^{\varepsilon} x_{1}(k)\right)\right\}, \\
& x_{2}(k+1) \geq x_{2}(k) \exp \left\{\left(r_{2}^{l}-b_{2}^{u}\left(M_{1}+\varepsilon_{1}\right)-c_{2}^{u}\left(U_{2}+\varepsilon_{1}\right)\right)\right. \\
&\left.\cdot\left(1-D_{2}^{\varepsilon} x_{2}(k)\right)\right\},
\end{aligned}
$$

where

$$
\begin{aligned}
& D_{1}^{\varepsilon}=\frac{a_{1}^{u}}{\left(r_{1}^{l}-b_{1}^{u}\left(M_{2}+\varepsilon_{1}\right)-\gamma^{u}\left(M_{1}+\varepsilon_{1}\right)\left(M_{2}+\varepsilon_{1}\right)^{2}-c_{1}^{u}\left(U_{1}+\varepsilon_{1}\right)\right)}, \\
& D_{2}^{\varepsilon}=\frac{a_{2}^{u}}{\left(r_{2}^{l}-b_{2}^{u}\left(M_{1}+\varepsilon_{1}\right)-c_{2}^{u}\left(U_{2}+\varepsilon_{1}\right)\right)} .
\end{aligned}
$$

Notice that

$$
\begin{array}{r}
\frac{a_{i}^{u}}{a_{i}^{l}} \geq 1, \\
\frac{\exp \left(r_{1}^{u}-1\right)}{r_{1}^{l}-b_{1}^{u}\left(M_{2}+\varepsilon_{1}\right)-\gamma^{u}\left(M_{1}+\varepsilon_{1}\right)\left(M_{2}+\varepsilon_{1}\right)^{2}-c_{1}^{u}\left(U_{1}+\varepsilon_{1}\right)}>1, \\
\frac{\exp \left(r_{2}^{u}-1\right)}{r_{2}^{l}-b_{2}^{u}\left(M_{1}+\varepsilon_{1}\right)-c_{2}^{u}\left(U_{2}+\varepsilon_{1}\right)}>1,
\end{array}
$$

then

$$
\begin{aligned}
& D_{1}^{\varepsilon} \cdot M_{1}=\frac{a_{1}^{u}}{r_{1}^{l}-b_{1}^{u}\left(M_{2}+\varepsilon_{1}\right)-\gamma^{u}\left(M_{1}+\varepsilon_{1}\right)\left(M_{2}+\varepsilon_{1}\right)^{2}-c_{1}^{u}\left(U_{1}+\varepsilon_{1}\right)} \cdot \frac{1}{a_{1}^{l}} \exp \left(r_{1}^{u}-1\right)>1, \\
& D_{2}^{\varepsilon} \cdot M_{2}=\frac{a_{2}^{u}}{r_{2}^{l}-b_{2}^{u}\left(M_{1}+\varepsilon_{1}\right)-c_{2}^{u}\left(U_{2}+\varepsilon_{1}\right)} \cdot \frac{1}{a_{2}^{l}} \exp \left(r_{2}^{u}-1\right)>1 .
\end{aligned}
$$

Hence, according to Lemma 4, we have

$$
\begin{aligned}
& \liminf _{k \longrightarrow+\infty} x_{1}(k) \geq \frac{1}{D_{1}^{\varepsilon}} \cdot \exp \left(r_{1}^{l}-b_{1}^{u}\left(M_{2}+\varepsilon_{1}\right)-\gamma^{u}\left(M_{1}+\varepsilon_{1}\right)\left(M_{2}+\varepsilon_{1}\right)^{2}-c_{1}^{u}\left(U_{1}+\varepsilon_{1}\right)-a_{1}^{u} M_{1}\right), \\
& \liminf _{k \longrightarrow+\infty} x_{2}(k) \geq \frac{1}{D_{2}^{\varepsilon}} \cdot \exp \left(r_{2}^{l}-b_{2}^{u}\left(M_{1}+\varepsilon_{1}\right)-c_{2}^{u}\left(U_{2}+\varepsilon_{1}\right)-a_{2}^{u} M_{2}\right) .
\end{aligned}
$$

Setting $\varepsilon_{1} \longrightarrow 0$, it follows that

$$
\begin{aligned}
& \liminf _{k \longrightarrow+\infty} x_{1}(k) \geq \frac{1}{D_{1}} \cdot \exp \left(r_{1}^{l}-b_{1}^{u} M_{2}-\gamma^{u} M_{1} M_{2}^{2}-c_{1}^{u} U_{1}-a_{1}^{u} M_{1}\right) \stackrel{\text { def }}{=} m_{1}, \\
& \liminf _{k \longrightarrow+\infty} x_{2}(k) \geq \frac{1}{D_{2}} \cdot \exp \left(r_{2}^{l}-b_{2}^{u} M_{1}-c_{2}^{u} U_{2}-a_{2}^{u} M_{2}\right) \stackrel{\text { def }}{=} m_{2},
\end{aligned}
$$


where

$$
\begin{aligned}
& D_{1}=\frac{a_{1}^{u}}{\left(r_{1}^{l}-b_{1}^{u} M_{2}-\gamma^{u} M_{1} M_{2}^{2}-c_{1}^{u} U_{1}\right)}, \\
& D_{2}=\frac{a_{2}^{u}}{\left(r_{2}^{l}-b_{2}^{u} M_{1}-c_{2}^{u} U_{2}\right)} .
\end{aligned}
$$

According to Lemma 5, from (35) and (36), we have, for any $\varepsilon_{2}>0$ small enough (without loss of generality, assume that $\left.\varepsilon_{2}<(1 / 2) \min _{i}\left\{m_{i}\right\}, i=1,2\right)$, there exists $N_{3}>N_{2}$, such that

$$
\sum_{s=0}^{\infty} L_{i}(s) x_{i}(k-s) \geq m_{i}-\varepsilon_{2}, \quad \text { for all } n \geq N_{3} .
$$

For $n \geq N_{3}$, from (38) and the third and fourth equations of system (5), we have

$$
u_{i}(k+1) \geq\left(1-e_{i}^{u}\right) u_{i}(k)+d_{i}^{l}\left(m_{i}-\varepsilon_{2}\right) .
$$

Hence, by applying Lemmas 1 and 2 to (39), we obtain

$$
\limsup _{k \longrightarrow+\infty} u_{i}(k) \geq \frac{d_{i}^{l}\left(m_{i}-\varepsilon_{2}\right)}{e_{i}^{u}} \text {. }
$$

Setting $\varepsilon_{2} \longrightarrow 0$, it follows that

$$
\underset{k \longrightarrow+\infty}{\limsup } u_{i}(k) \geq \frac{d_{i}^{l} m_{i}}{e_{i}^{u}} \stackrel{\text { def }}{=} B_{i} .
$$

This ends the proof of Theorem 3.1.

\section{Extinction}

Concerned with the extinction property of system (5), we could establish the following results.

Theorem 2. Assume that

$$
\frac{r_{1}^{u}}{r_{2}^{l}}<\min \left\{\frac{a_{1}^{l} e_{1}^{u}+c_{1}^{l} d_{1}^{l}}{b_{2}^{u} e_{1}^{u}}, \frac{b_{1}^{l} e_{2}^{l}}{\left(1+M_{2}\right)\left(a_{2}^{u} e_{2}^{l}+c_{2}^{u} d_{2}^{u}\right)}\right\}(\mathrm{H} 1),
$$

holds. Let $\left(x_{1}(k), x_{2}(k), u_{1}(k), u_{2}(k)\right)$ be any positive solution of system (5), then

$$
\begin{aligned}
& \lim _{k \longrightarrow+\infty} x_{1}(k)=0, \\
& \lim _{k \longrightarrow+\infty} u_{1}(k)=0 .
\end{aligned}
$$

Theorem 3. Assume that

$$
\begin{aligned}
& \frac{r_{1}^{l}}{r_{2}^{u}>\max }\left\{\left(1+M_{1}\right) \frac{a_{1}^{u} e_{1}^{l}+c_{1}^{u} d_{1}^{u}}{b_{2}^{l} e_{1}^{l}}, \frac{b_{1}^{u} e_{2}^{u}}{a_{2}^{l} e_{2}^{u}+c_{2}^{l} d_{2}^{l}}\right\}(\mathrm{H} 2) \\
& \gamma^{u}<\min \frac{1}{M_{1} M_{2}^{2}}\left\{r_{1}^{l}-\left(1+M_{1}\right) r_{2}^{u} \frac{a_{1}^{u} e_{1}^{l}+c_{1}^{u} d_{1}^{u}}{b_{2}^{l} e_{1}^{l}}, r_{1}^{l}\right. \\
& \left.\quad-r_{2}^{u} \frac{b_{1}^{u} e_{2}^{u}}{a_{2}^{l} e_{2}^{u}+c_{2}^{l} d_{2}^{l}}\right\} \text { (H3) }
\end{aligned}
$$

hold. Let $\left(x_{1}(k), x_{2}(k), u_{1}(k), u_{2}(k)\right)$ be any positive solution of system (5), then

$$
\lim _{k \longrightarrow+\infty} x_{2}(k)=0, \quad \lim _{k \longrightarrow+\infty} u_{2}(k)=0 .
$$

Proof of Theorem 2. Condition $\left(H_{1}\right)$ is equivalent to

$$
\begin{aligned}
& \frac{c_{1}^{l}}{e_{1}^{u}}>\frac{r_{1}^{u}}{r_{2}^{l}} \frac{b_{2}^{u}}{d_{1}^{l}}-\frac{a_{1}^{l}}{d_{1}^{l}}, \\
& \frac{c_{2}^{u}}{e_{2}^{l}}<\frac{r_{2}^{l} b_{1}^{l}}{\left(1+M_{2}\right) r_{1}^{u} d_{2}^{u}}-\frac{a_{2}^{u}}{d_{2}^{u}} .
\end{aligned}
$$

From (46), one could choose positive constants $\alpha, \beta$, $\eta_{1}$, and $\eta_{2}$ and enough small positive $\varepsilon$ such that

$$
\frac{r_{1}^{u}}{r_{2}^{l}}<\frac{\beta}{\alpha}
$$$$
\frac{c_{1}^{l}}{e_{1}^{u}}>\frac{\eta_{1}}{\alpha}>\frac{\beta b_{2}^{u}-\alpha a_{1}^{l}}{\alpha d_{1}^{l}}>\frac{r_{1}^{u}}{r_{2}^{l}} \frac{b_{2}^{u}}{d_{1}^{l}}-\frac{a_{1}^{l}}{d_{1}^{l}},
$$

$$
\frac{c_{2}^{u}}{e_{2}^{l}}<\frac{\eta_{2}}{\beta}<\frac{\alpha b_{1}^{l}-\left(1+M_{2}+\varepsilon\right) \beta a_{2}^{u}}{\left(1+M_{2}+\varepsilon\right) \beta d_{2}^{u}}
$$

$$
<\frac{r_{2}^{l} b_{1}^{l}}{\left(1+M_{2}+\varepsilon\right) r_{1}^{u} d_{2}^{u}}-\frac{a_{2}^{u}}{d_{2}^{u}}
$$

That is,

$$
\begin{gathered}
\alpha r_{1}^{u}-\beta r_{2}^{l}=-\lambda_{1}<0, \\
\eta_{1} e_{1}^{u}-\alpha c_{1}^{l}<0, \\
\beta c_{2}^{u}-\eta_{2} e_{2}^{l}<0, \\
\beta b_{2}^{u}-\alpha a_{1}^{l}-\eta_{1} d_{1}^{l}<0, \\
\eta_{2} d_{2}^{u}+\beta a_{2}^{u}-\frac{\alpha b_{1}^{l}}{1+M_{2}+\varepsilon}<0 .
\end{gathered}
$$

Let $\left(x_{1}(k), x_{2}(k), u_{1}(k), u_{2}(k)\right)^{T}$ be a positive solution of system (5). For above $\varepsilon$, from Theorem 1 , there exists a enough large $T_{1}$, such that

$$
\begin{array}{r}
x_{i}(t)<M_{i}+\varepsilon, \\
u_{i}(t)<U_{i}+\varepsilon, \\
t \geq T_{1}, \\
i=1,2 .
\end{array}
$$

Consider the following discrete Lyapunov functional 


$$
\begin{aligned}
V_{1}(k)= & x_{1}^{\alpha}(k) x_{2}^{-\beta}(k) \exp \left\{\eta_{2} u_{2}(k)-\eta_{1} u_{1}(k)-\frac{\alpha \sum_{s=0}^{\infty} K_{12}(s) \sum_{q=k-s}^{k-1} b_{1}(q+s) x_{2}(q)}{1+M_{2}}-\alpha \sum_{s=0}^{\infty} H_{1}(s) \sum_{q=k-s}^{k-1} c_{1}(q+s) u_{1}(q)\right. \\
& +\frac{\beta \sum_{s=0}^{\infty} K_{21}(s) \sum_{q=k-s}^{k-1} b_{2}(q+s) x_{1}(q)}{1+m_{1}}+\beta \sum_{s=0}^{\infty} H_{2}(s) \sum_{q=k-s}^{k-1} c_{2}(q+s) u_{2}(q)+\eta_{2} \sum_{s=0}^{\infty} L_{2}(s) \sum_{q=k-s}^{k-1} d_{2}(q+s) x_{2}(q) \\
& \left.-\eta_{1} \sum_{s=0}^{\infty} L_{1}(s) \sum_{q=k-s}^{k-1} d_{1}(q+s) x_{1}(q)\right\} .
\end{aligned}
$$

By calculating, we obtain

$$
\begin{aligned}
& \frac{V_{1}(k+1)}{V_{1}(k)}=\exp \left\{\alpha\left(r_{1}(k)-a_{1}(k) x_{1}(k)-\frac{b_{1}(k) \sum_{s=0}^{\infty} K_{12}(s) x_{2}(k-s)}{1+\sum_{s=0}^{\infty} K_{12}(s) x_{2}(k-s)}-\gamma(k) x_{1}(k) x_{2}^{2}(k)-c_{1}(k) \sum_{s=0}^{\infty} H_{1}(s) u_{1}(k-s)\right)\right. \\
& -\beta\left(r_{2}(k)-\frac{b_{2}(k) \sum_{s=0}^{\infty} K_{21}(s) x_{1}(k-s)}{1+\sum_{s=0}^{\infty} K_{21}(s) x_{1}(k-s)}-a_{2}(k) x_{2}(k)-c_{2}(k) \sum_{s=0}^{\infty} H_{2}(s) u_{2}(k-s)\right) \\
& -\eta_{1}\left(-e_{1}(k) u_{1}(k)+d_{1}(k) \sum_{s=0}^{\infty} L_{1}(s) x_{1}(k-s)\right)+\eta_{2}\left(-e_{2}(k) u_{2}(k)+d_{2}(k) \sum_{s=0}^{\infty} L_{2}(s) x_{2}(k-s)\right) \\
& -\frac{\alpha}{1+M_{2}} \sum_{s=0}^{\infty} K_{12}(s)\left(b_{1}(k+s) x_{2}(k)-b_{1}(k) x_{2}(k-s)\right)-\alpha \sum_{s=0}^{\infty} H_{1}(s)\left(c_{1}(k+s) u_{1}(k)-c_{1}(k) u_{1}(k-s)\right) \\
& +\frac{\beta}{1+m_{1}} \sum_{s=0}^{\infty} K_{21}(s)\left(b_{2}(k+s) x_{1}(k)-b_{2}(k) x_{1}(k-s)\right)+\beta \sum_{s=0}^{\infty} H_{2}(s)\left(c_{2}(k+s) u_{2}(k)-c_{2}(k) u_{2}(k-s)\right) \\
& \left.-\eta_{1} \sum_{s=0}^{\infty} L_{1}(s)\left(d_{1}(k+s) x_{1}(k)-d_{1}(k) x_{1}(k-s)\right)+\eta_{2} \sum_{s=0}^{\infty} L_{2}(s)\left(d_{2}(k+s) x_{2}(k)-d_{2}(k) x_{2}(k-s)\right)\right\} \\
& \leq \exp \left\{\left(\alpha r_{1}(k)-\beta r_{2}(k)\right)+\left(-\alpha a_{1}(k)+\beta \sum_{s=0}^{\infty} K_{21}(s) b_{2}(k+s)-\eta_{1} \sum_{s=0}^{\infty} L_{1}(s) d_{1}(k+s)\right) x_{1}(k)\right. \\
& +\left(\beta a_{2}(k)+\eta_{2} \sum_{s=0}^{\infty} L_{2}(s) d_{2}(k+s)-\frac{\alpha \sum_{s=0}^{\infty} K_{12}(s) b_{1}(k+s)}{1+\left(M_{2}+\varepsilon\right)}\right) x_{2}(k)+\left(\eta_{1} e_{1}(k)-\alpha \sum_{s=0}^{\infty} H_{1}(s) c_{1}(k+s)\right) u_{1}(k) \\
& \left.+\left(\beta \sum_{s=0}^{\infty} H_{2}(s) c_{2}(k+s)-\eta_{2} e_{2}(k)\right) u_{2}(k)\right\} \\
& \leq \exp \left\{\left(\alpha r_{1}^{u}-\beta r_{2}^{l}\right)+\left(\beta b_{2}^{u}-\alpha a_{1}^{l}-\eta_{1} d_{1}^{l}\right) x_{1}(k)+\left(\eta_{2} d_{2}^{u}+\beta a_{2}^{u}-\frac{\alpha b_{1}^{l}}{1+\left(M_{2}+\varepsilon\right)}\right) x_{2}(k)\right. \\
& \left.+\left(\eta_{1} e_{1}^{u}-\alpha c_{1}^{l}\right) u_{1}(k)+\left(\beta c_{2}^{u}-\eta_{2} e_{2}^{l}\right) u_{2}(k)\right\} .
\end{aligned}
$$


From inequalities (48) and (51), we can obtain

$$
V_{1}(k+1) \leq V_{1}(k) \exp \left(-\lambda_{1}\right) .
$$

Therefore,

$$
V_{1}(k) \leq V_{1}(0) \exp \left(-k \lambda_{1}\right) .
$$

From (22) and (27), we know that there exists a $M>0$ such that

$$
\begin{aligned}
& x_{i}(k)<M, \\
& u_{i}(k)<M, \quad \text { for all } k \in Z, i=1,2 .
\end{aligned}
$$

Hence,

$$
\begin{aligned}
V_{1}(0)= & x_{1}^{\alpha}(0) x_{2}^{-\beta}(0) \exp \left\{\eta_{2} u_{2}(0)-\eta_{1} u_{1}(0)-\frac{\alpha \sum_{s=0}^{\infty} K_{12}(s) \sum_{q=-s}^{-1} b_{1}(q+s) x_{2}(q)}{1+M_{2}}-\alpha \sum_{s=0}^{\infty} H_{1}(s) \sum_{q=-s}^{-1} c_{1}(q+s) u_{1}(q)\right. \\
& +\frac{\beta \sum_{s=0}^{\infty} K_{21}(s) \sum_{q=-s}^{-1} b_{2}(q+s) x_{1}(q)}{1+m_{1}}+\beta \sum_{s=0}^{\infty} H_{2}(s) \sum_{q=-s}^{-1} c_{2}(q+s) u_{2}(q)+\eta_{2} \sum_{s=0}^{\infty} L_{2}(s) \sum_{q=-s}^{-1} d_{2}(q+s) x_{2}(q) \\
& \left.-\eta_{1} \sum_{s=0}^{\infty} L_{1}(s) \sum_{q=-s}^{-1} d_{1}(q+s) x_{1}(q)\right\} \\
< & x_{1}^{\alpha}(0) x_{2}^{-\beta}(0) \exp \left\{\eta_{2} M+\frac{\beta b_{2}^{u} M \sum_{s=0}^{\infty} K_{21}(s) s}{1+m_{1}}+\beta c_{2}^{u} M \sum_{s=0}^{\infty} H_{2}(s) s+\eta_{2} d_{2}^{u} M \sum_{s=0}^{\infty} L_{2}(s) s\right\} \\
= & x_{1}^{\alpha}(0) x_{2}^{-\beta}(0) \exp \left\{M\left(\eta_{2}+\frac{\beta b_{2}^{u} M \Theta_{21}}{1+m_{1}}+\beta c_{2}^{u} M \Lambda_{2}+\eta_{2} d_{2}^{u} M \Upsilon_{2}\right)\right\} \\
< & +\infty .
\end{aligned}
$$

On the contrary, we also have

$$
\begin{aligned}
V_{1}(k) \geq & x_{1}^{\alpha}(k) x_{2}^{-\beta}(k) \exp \left\{-\eta_{1} u_{1}(k)-\frac{\alpha \sum_{s=0}^{\infty} K_{12}(s) \sum_{q=k-s}^{k-1} b_{1}(q+s) x_{2}(q)}{1+M_{2}}-\alpha \sum_{s=0}^{\infty} H_{1}(s) \sum_{q=k-s}^{k-1} c_{1}(q+s) u_{1}(q)\right. \\
& \left.-\eta_{1} \sum_{s=0}^{\infty} L_{1}(s) \sum_{q=k-s}^{k-1} d_{1}(q+s) x_{1}(q)\right\} \\
\geq & x_{1}^{\alpha}(k) M^{-\beta} \exp \left\{-\eta_{1} M-\frac{\alpha b_{1}^{u} M \sum_{s=0}^{\infty} K_{12}(s) s}{1+M_{2}}-\alpha c_{1}^{u} M \sum_{s=0}^{\infty} H_{1}(s) s-\eta_{1} d_{1}^{u} M \sum_{s=0}^{\infty} L_{1}(s) s\right\} \\
= & x_{1}^{\alpha}(k) M^{-\beta} \exp \left\{-\eta_{1}\left(M+d_{1}^{u} M \Upsilon_{1}\right)-\alpha\left(\frac{b_{1}^{u} M \Theta_{12}}{1+M_{2}}+c_{1}^{u} M \Lambda_{1}\right)\right\} .
\end{aligned}
$$

Combining inequalities (53), (55), and (56), we have

$$
x_{1}(k) \leq \Delta \exp \left\{-\frac{\lambda_{1}}{\alpha} k\right\},
$$

where

$$
\begin{aligned}
\Delta= & \left(V_{1}(0)\right)^{1 / \alpha} M^{\beta / \alpha} \exp \left\{\frac{\eta_{1}}{\alpha}\left(M+d_{1}^{u} M \Upsilon_{1}\right)\right. \\
& \left.+\left(\frac{b_{1}^{u} M \Theta_{12}}{1+M_{2}}+c_{1}^{u} M \Lambda_{1}\right)\right\}<+\infty .
\end{aligned}
$$




$$
\lim _{k \longrightarrow+\infty} x_{1}(k)=0
$$

Similar to the corresponding proof of Theorem 1 by Chen et al. [7], we can easily obtain that $\lim _{k \longrightarrow+\infty} u_{1}(k)=0$. This ends the proof of Theorem 2 .

Proof of Theorem 3. Condition $\left(\mathrm{H}_{2}\right)$ is equivalent to

$$
\begin{aligned}
& \frac{c_{1}^{u}}{e_{1}^{l}}<\frac{r_{1}^{l} b_{2}^{l}}{\left(1+M_{1}\right) r_{2}^{u} d_{1}^{u}}-\frac{a_{1}^{u}}{d_{1}^{u}}, \\
& \frac{c_{2}^{l}}{e_{2}^{u}}>\frac{r_{2}^{u}}{r_{1}^{l}} \frac{b_{1}^{u}}{d_{2}^{l}}-\frac{a_{2}^{l}}{d_{2}^{l}} .
\end{aligned}
$$

From $\left(\mathrm{H}_{3}\right)$ and (60), there exist positive constants $\alpha, \beta, \delta_{1}$, and $\delta_{2}$ and enough small positive $\varepsilon$ such that

$$
\begin{gathered}
\frac{r_{1}^{l}}{r_{2}^{u}}>\frac{\beta}{\alpha}, \\
\frac{c_{1}^{u}}{e_{1}^{l}}<\frac{\delta_{1}}{\alpha}<\frac{\beta b_{2}^{l}-\left(1+M_{1}+\varepsilon\right) \alpha a_{1}^{u}}{\left(1+M_{1}+\varepsilon\right) \alpha d_{1}^{u}}<\frac{r_{1}^{l} b_{2}^{l}}{\left(1+M_{1}+\varepsilon\right) r_{2}^{u} d_{1}^{u}}-\frac{a_{1}^{u}}{d_{1}^{u}}, \\
\frac{c_{2}^{l}}{e_{2}^{u}}>\frac{\delta_{2}}{\beta}>\frac{\alpha b_{1}^{u}-\beta a_{2}^{l}}{\beta d_{2}^{l}}>\frac{r_{2}^{u}}{r_{1}^{l}} \frac{b_{1}^{u}}{d_{2}^{l}}-\frac{a_{2}^{l}}{d_{2}^{l}}, \\
\frac{\left(1+M_{1}+\varepsilon\right)\left(a_{1}^{u} e_{1}^{l}+c_{1}^{u} d_{1}^{u}\right)}{b_{2}^{l} e_{1}^{l}}<\frac{\beta}{\alpha}<\frac{r_{1}^{l}-\gamma^{u}\left(M_{1}+\varepsilon\right)\left(M_{2}+\varepsilon\right)^{2}}{r_{2}^{u}}, \\
\frac{b_{1}^{u} e_{2}^{u}}{a_{2}^{l} e_{2}^{u}+c_{2}^{l} d_{2}^{l}}<\frac{\beta}{\alpha}<\frac{r_{1}^{l}-\gamma^{u}\left(M_{1}+\varepsilon\right)\left(M_{2}+\varepsilon\right)^{2}}{r_{2}^{u}} .
\end{gathered}
$$

That is,

$$
\begin{gathered}
\alpha c_{1}^{u}-\delta_{1} e_{1}^{l}<0, \\
\delta_{2} e_{2}^{u}-\beta c_{2}^{l}<0, \\
\alpha a_{1}^{u}-\frac{\beta b_{2}^{l}}{1+M_{1}+\varepsilon}+\delta_{1} d_{1}^{u}<0, \\
\alpha b_{1}^{u}-\beta a_{2}^{l}-\delta_{2} d_{2}^{l}<0, \\
-\alpha r_{1}^{l}+\beta r_{2}^{u}+\alpha \gamma^{u}\left(M_{1}+\varepsilon\right)\left(M_{2}+\varepsilon\right)^{2}=-\xi_{1}<0 .
\end{gathered}
$$

Let $\left(x_{1}(k), x_{2}(k), u_{1}(k), u_{2}(k)\right)^{T}$ be a positive solution of system (5). For above $\varepsilon$, from Theorem 1, there exists an enough large $T_{2}$, such that

$$
V_{2}(k)=x_{1}^{-\alpha}(k) x_{2}^{\beta}(k) \exp \left\{\delta_{1} u_{1}(k)-\delta_{2} u_{2}(k)\right.
$$$$
+\alpha \sum_{s=0}^{\infty} K_{12}(s) \sum_{q=k-s}^{k-1} b_{1}(q+s) x_{2}(q)
$$$$
+\alpha \sum_{s=0}^{\infty} H_{1}(s) \sum_{q=k-s}^{k-1} c_{1}(q+s) u_{1}(q)
$$$$
-\frac{\beta \sum_{s=0}^{\infty} K_{21}(s) \sum_{q=k-s}^{k-1} b_{2}(q+s) x_{1}(q)}{1+M_{1}}
$$

$$
x_{i}(k)<M_{i}+\varepsilon, u_{i}(k)<U_{i}+\varepsilon, t \geq T_{2}, \quad i=1,2 .
$$

Define the following Lyapunov functional as follows:

$$
\begin{aligned}
& -\beta \sum_{s=0}^{\infty} H_{2}(s) \sum_{q=k-s}^{k-1} c_{2}(q+s) u_{2}(q) \\
& -\delta_{2} \sum_{s=0}^{\infty} L_{2}(s) \sum_{q=k-s}^{k-1} d_{2}(q+s) x_{2}(q) \\
& \left.+\delta_{1} \sum_{s=0}^{\infty} L_{1}(s) \sum_{q=k-s}^{k-1} d_{1}(q+s) x_{1}(q)\right\} .
\end{aligned}
$$


Similar to the analysis of (51)-(58), we have

$$
\lim _{k \longrightarrow+\infty} x_{2}(k)=0, \lim _{k \longrightarrow+\infty} u_{2}(k)=0 .
$$

This ends the proof of Theorem 3.
From Theorems 2 and 3, we know that under some suitable assumption, one of the species in the system may be driven to extinction; in this case, one interesting problem is to investigate the stability property of the rest species.

Consider the following discrete equations:

$$
\begin{aligned}
& x_{2}(k+1)=x_{2}(k) \exp \left\{r_{2}(k)-a_{2}(k) x_{2}(k)-c_{2}(k) \sum_{s=0}^{\infty} H_{2}(s) u_{2}(k-s)\right\}, \\
& u_{2}(k+1)=u_{2}(k)\left(1-e_{2}(k)\right)+d_{2}(k) \sum_{s=0}^{\infty} L_{2}(s) x_{2}(k-s) . \\
& x_{1}(k+1)=x_{1}(k) \exp \left\{r_{1}(k)-a_{1}(k) x_{1}(k)-c_{1}(k) \sum_{s=0}^{\infty} H_{1}(s) u_{1}(k-s)\right\}, \\
& u_{1}(k+1)=u_{1}(k)\left(1-e_{1}(k)\right)+d_{1}(k) \sum_{s=0}^{\infty} L_{1}(s) x_{1}(k-s) .
\end{aligned}
$$

Theorem 4. Assume that $\left(H_{1}\right)$ holds; also,

$$
c_{2}^{u}<\frac{A_{22} e_{2}^{l}}{d_{2}^{u}},
$$

holds; then, for any positive solution $\left(x_{1}(k), x_{2}(k), u_{1}(k)\right.$, $\left.u_{2}(k)\right)$ of system (5) and any positive solution $\left(x_{2}^{*}(k), u_{2}^{*}(k)\right)$ of system (66), we have

$$
\begin{aligned}
\lim _{k \longrightarrow+\infty} x_{1}(k) & =0, \\
\lim _{k \longrightarrow+\infty} u_{1}(k) & =0, \\
\lim _{k \longrightarrow+\infty}\left(x_{2}(k)-x_{2}^{*}(k)\right) & =0, \\
\lim _{k \longrightarrow+\infty}\left(u_{2}(k)-u_{2}^{*}(k)\right) & =0,
\end{aligned}
$$

where $A_{22}=\min \left\{a_{2}^{l},\left(2 / M_{2}\right)-a_{2}^{u}\right\}$.

Theorem 5. Assume that $\left(\mathrm{H}_{2}\right)$ and $\left(\mathrm{H}_{3}\right)$ hold; also,

$$
c_{1}^{u}<\frac{A_{11} e_{1}^{l}}{d_{1}^{u}},
$$

holds; then, for any positive solution $\left(x_{1}(k), x_{2}(k), u_{1}(k)\right.$, $\left.u_{2}(k)\right)$ of system (5) and any positive solution $\left(x_{1}^{*}(k), u_{1}^{*}(k)\right)$ of system (67), we have

$$
\begin{aligned}
\lim _{k \longrightarrow+\infty}\left(x_{1}(k)-x_{1}^{*}(k)\right) & =0, \\
\lim _{k \longrightarrow+\infty}\left(u_{1}(k)-u_{1}^{*}(k)\right) & =0, \\
\lim _{k \longrightarrow+\infty} x_{2}(k) & =0, \\
\lim _{k \longrightarrow+\infty} u_{2}(k) & =0,
\end{aligned}
$$

where $A_{11}=\min \left\{a_{1}^{l},\left(2 / M_{1}\right)-a_{1}^{u}\right\}$.

Proof of Theorem 4. By conditions (68), we can choose positive constants $\rho_{1}$ and $\rho_{2}$ such that

$$
\frac{e_{2}^{l}}{c_{2}^{u}}>\frac{\rho_{1}}{\rho_{2}}>\frac{d_{2}^{u}}{A_{22}} .
$$

Thus, there exists enough small positive constant $\omega$ and $\varepsilon$ such that

$$
\begin{gathered}
\rho_{1} A_{22}^{\varepsilon}-\rho_{2} d_{2}^{u}>\omega, \\
\rho_{2} e_{2}^{l}-\rho_{1} c_{2}^{u}>\omega,
\end{gathered}
$$

where $A_{22}^{\varepsilon}=\min \left\{a_{2}^{l},\left(2 /\left(M_{2}+\varepsilon\right)\right)-a_{2}^{u}\right\}$.

Now, we define a Lyapunov functional as follows:

$$
\begin{aligned}
G_{1}(k)= & \rho_{1}\left[\left|\ln x_{2}(k)-\ln x_{2}^{*}(k)\right|\right. \\
& +\sum_{s=0}^{\infty} K_{21}(s) \sum_{q=n-s}^{n-1} b_{2}(q+s) x_{1}(q) \\
& \left.+\sum_{s=0}^{\infty} H_{2}(s) \sum_{q=n-s}^{n-1} c_{2}(q+s)\left|u_{2}(q)-u_{2}^{*}(q)\right|\right] \\
& +\rho_{2}\left[\left|u_{2}(k)-u_{2}^{*}(k)\right|\right. \\
& \left.+\sum_{s=0}^{\infty} L_{2}(s) \sum_{q=n-s}^{n-1} d_{2}(q+s)\left|x_{2}(q)-x_{2}^{*}(q)\right|\right] .
\end{aligned}
$$

One could easily see that $G_{1}(k) \geq 0$ for all $k \in Z^{+}$. Also, for any fixed $k^{*} \in Z^{+}$, from (54), one could see that

$$
\begin{aligned}
G_{1}\left(k^{*}\right)= & \rho_{1}\left|\ln x_{2}\left(k^{*}\right)-\ln x_{2}^{*}\left(k^{*}\right)\right|+\rho_{1} M b_{2}^{u} \sum_{s=0}^{\infty} K_{21}(s) s \\
& +\rho_{1} c_{2}^{u} \sup _{q \in Z^{+}, q \leq k^{*}}\left|u_{2}(q)-u_{2}^{*}(q)\right| \sum_{s=0}^{\infty} H_{2}(s) s \\
& +\rho_{2}\left|u_{2}\left(k^{*}\right)-u_{2}^{*}\left(k^{*}\right)\right| \\
& +\rho_{2} d_{2}^{u} \sup _{q \in Z^{+}, q \leq k^{*}}\left|x_{2}(q)-x_{2}^{*}(q)\right| \sum_{s=0}^{\infty} L_{2}(s) s \\
< & +\infty .
\end{aligned}
$$


It follows from the second equation of system (5) and the mean value theorem that

$$
\begin{aligned}
\triangle G_{1}(k) \leq & \rho_{1}\left[-\left(\frac{1}{\varphi_{2}(k)}-\left|\frac{1}{\varphi_{2}(k)}-a_{2}(k)\right|\right)\left|x_{2}(k)-x_{2}^{*}(k)\right|+b_{2}^{u} x_{1}(k)+c_{2}^{u}\left|u_{2}(k)-u_{2}^{*}(k)\right|\right] \\
& +\rho_{2}\left[-e_{2}^{l}\left|u_{2}(k)-u_{2}^{*}(k)\right|+d_{2}^{u}\left|x_{2}(k)-x_{2}^{*}(k)\right|\right] . \\
\leq & -\left\{\rho_{1} \min \left[a_{2}^{l}, \frac{2}{M_{2}+\varepsilon}-a_{2}^{u}\right]-\rho_{2} d_{2}^{u}\right\}\left|x_{2}(k)-x_{2}^{*}(k)\right|-\left\{\rho_{2} e_{2}^{l}-\rho_{1} c_{2}^{u}\right\}\left|u_{2}(k)-u_{2}^{*}(k)\right|+\rho_{1} b_{2}^{u} x_{1}(k) \\
= & -\left\{\rho_{1} A_{22}^{\varepsilon}-\rho_{2} d_{2}^{u}\right\}\left|x_{2}(k)-x_{2}^{*}(k)\right|-\left\{\rho_{2} e_{2}^{l}-\rho_{1} c_{2}^{u}\right\}\left|u_{2}(k)-u_{2}^{*}(k)\right|+\rho_{1} b_{2}^{u} x_{1}(k) \\
\leq & -\omega\left(\left|x_{2}(k)-x_{2}^{*}(k)\right|+\left|u_{2}(k)-u_{2}^{*}(k)\right|\right)+\rho x_{1}(k)
\end{aligned}
$$

where $\rho=\rho_{1} b_{2}^{u}$.

Summating both sides of the above inequality from $k^{*}$ to $k$, we have

$$
\begin{aligned}
\sum_{p=k^{*}}^{k}\left(G_{1}(p+1)-G_{1}(p)\right) \leq & -\omega \sum_{p=k^{*}}^{k}\left(\left|x_{2}(p)-x_{2}^{*}(p)\right|\right. \\
& \left.+\left|u_{2}(p)-u_{2}^{*}(p)\right|\right)+\rho \sum_{p=k^{*}}^{k} x_{1}(p) .
\end{aligned}
$$

Hence,

$$
\begin{aligned}
& G_{1}(k+1)+\omega \sum_{p=k^{*}}^{k}\left(\left|x_{2}(p)-x_{2}^{*}(p)\right|+\left|u_{2}(p)-u_{2}^{*}(p)\right|\right) \\
& \quad \leq G_{1}\left(k^{*}\right)+\rho \sum_{p=k^{*}}^{k} x_{1}(p) .
\end{aligned}
$$

Then, from (75) and (57), we have

$$
\begin{gathered}
\sum_{p=k^{*}}^{k}\left(\left|x_{2}(p)-x_{2}^{*}(p)\right|+\left|u_{2}(p)-u_{2}^{*}(p)\right|\right) \\
\leq \frac{G_{1}\left(k^{*}\right)+\rho \sum_{p=k^{*}}^{k} x_{1}(p)}{\omega}<+\infty .
\end{gathered}
$$

Therefore,

$$
\sum_{p=k^{*}}^{\infty}\left(\left|x_{2}(p)-x_{2}^{*}(p)\right|+\left|u_{2}(p)-u_{2}^{*}(p)\right|\right)<+\infty
$$

which means that

$$
\lim _{k \longrightarrow+\infty}\left(\left|x_{2}(k)-x_{2}^{*}(k)\right|+\left|u_{2}(k)-u_{2}^{*}(k)\right|\right)=0 .
$$

Consequently,

$$
\begin{aligned}
& \lim _{k \longrightarrow+\infty}\left(x_{2}(k)-x_{2}^{*}(k)\right)=0, \\
& \lim _{k \longrightarrow+\infty}\left(u_{2}(k)-u_{2}^{*}(k)\right)=0 .
\end{aligned}
$$

This completes the proof of Theorem 4 .

Proof of Theorem 5. The proof of Theorem 5 is similar to that of Theorem 4, and we omit the detail here.

\section{Examples}

In this section, we shall give three examples to illustrate the feasibility of main results.

Example 1. Consider the following equations: 


$$
\begin{aligned}
& x_{1}(k+1)=x_{1}(k) \exp \left\{1-(5.2+0.2 \sin (k)) x_{1}(k)-\frac{0.5 \sum_{s=0}^{\infty}((e-1) / e) e^{-s} x_{2}(k-s)}{1+\sum_{s=0}^{\infty}((e-1) / e) e^{-s} x_{2}(k-s)}-3 x_{1}(k) x_{2}^{2}(k)-3 \sum_{s=0}^{\infty} \frac{e^{2}-1}{e^{2}} e^{-2 s} u_{1}(k-s)\right\} \\
& x_{2}(k+1)=x_{2}(k) \exp \left\{0.95-0.05 \cos (k)-\frac{0.2 \sum_{s=0}^{\infty}((e-1) / e) e^{-s} x_{1}(k-s)}{1+\sum_{s=0}^{\infty}((e-1) / e) e^{-s} x_{1}(k-s)}-(2.5+0.5 \sin (k)) x_{2}(k)-0.8 \sum_{s=0}^{\infty} \frac{e^{2}-1}{e^{2}} e^{-2 s} u_{2}(k-s)\right\} \\
& u_{1}(k+1)=u_{1}(k)(1-0.5)+0.25 \sum_{s=0}^{\infty} \frac{e^{3}-1}{e^{3}} e^{-3 s} x_{1}(k-s) \\
& u_{2}(k+1)=u_{2}(k)(1-0.8)+0.2 \sum_{s=0}^{\infty} \frac{e^{3}-1}{e^{3}} e^{-3 s} x_{2}(k-s) .
\end{aligned}
$$

One could easily see that conditions $\left(C_{1}\right)$ and $\left(C_{2}\right)$ are satisfied. Also, by calculating, one has

$$
\begin{aligned}
& M_{1}=\frac{1}{a_{1}^{l}} \exp \left(r_{1}^{u}-1\right)=0.2, \\
& M_{2}=\frac{1}{a_{2}^{l}} \exp \left(r_{2}^{u}-1\right)=0.5 \text {, } \\
& U_{1}=\frac{d_{1}^{u}}{e_{1}^{l}} M_{1}=0.1 \text {, } \\
& U_{2}=\frac{d_{2}^{u}}{e_{2}^{l}} M_{2}=\frac{1}{8}, \\
& r_{1}^{l}-b_{1}^{u} M_{2}-c_{1}^{u} U_{1}=0.45>0, \\
& r_{2}^{l}-b_{2}^{u} M_{1}-c_{2}^{u} U_{2}=0.4>0 \text {, } \\
& \gamma^{u}=3<\frac{r_{1}^{l}-b_{1}^{u} M_{2}-c_{1}^{u} U_{1}}{M_{1} M_{2}^{2}}=22.5 . \\
& m_{i} \leq \liminf _{k \longrightarrow+\infty} x_{i}(k)<\limsup _{k \longrightarrow+\infty} x_{i}(k) \leq M_{i}, \\
& B_{i} \leq \liminf _{k \longrightarrow+\infty} u_{i}(k)<\limsup _{k \longrightarrow+\infty} u_{i}(k) \leq U_{i} \text {, } \\
& x_{1}(k+1)=x_{1}(k) \exp \left\{1-(3.2+0.2 \sin (k)) x_{1}(k)-\frac{5 \sum_{s=0}^{\infty}((e-1) / e) e^{-s} x_{2}(k-s)}{1+\sum_{s=0}^{\infty}((e-1) / e) e^{-s} x_{2}(k-s)}-0.00005 x(k) x_{2}^{2}(k)-0.3 \sum_{s=0}^{\infty} \frac{e^{2}-1}{e^{2}} e^{-2 s} u_{1}(k-s)\right\} \text {, } \\
& x_{2}(k+1)=x_{2}(k) \exp \left\{0.95-0.05 \cos (k)-\frac{1.5 \sum_{s=0}^{\infty}((e-1) / e) e^{-s} x_{1}(k-s)}{1+\sum_{s=0}^{\infty}((e-1) / e) e^{-s} x_{1}(k-s)}-0.4 x_{2}(k)-0.1 \sum_{s=0}^{\infty} \frac{e^{2}-1}{e^{2}} e^{-2 s} u_{2}(k-s)\right\} \\
& u_{1}(k+1)=u_{1}(k)(1-0.9)+5 \sum_{s=0}^{\infty} \frac{e^{3}-1}{e^{3}} e^{-3 s} x_{1}(k-s) \\
& u_{2}(k+1)=u_{2}(k)(1-0.6)+2 \sum_{s=0}^{\infty} \frac{e^{3}-1}{e^{3}} e^{-3 s} x_{2}(k-s) .
\end{aligned}
$$

Clearly, condition (18) is satisfied, and so from Theorem 1 , we have

One could easily see that conditions $\left(C_{1}\right)$ and $\left(C_{2}\right)$ are satisfied. Also, by calculating, one has 


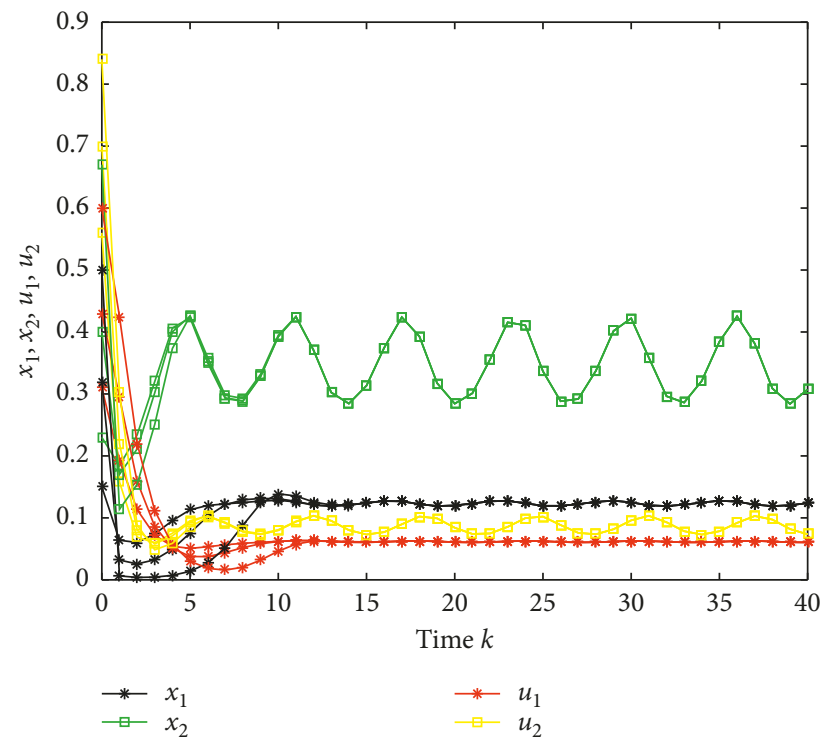

Figure 1: Numeric simulations of the solutions $\left(x_{1}(k), x_{2}(k), u_{1}(k), u_{2}(k)\right)$ of system (83), with the initial conditions $\left(x_{1}(s), x_{2}(s), u_{1}(s), u_{2}(s)\right)=(0.15,0.23,0.31,0.56),(0.32,0.4,0.43,0.7),(0.5,0.67,0.6,0.84), s=\ldots,-n,-n+1, \ldots,-1,0$, respectively.

$$
\begin{aligned}
\frac{r_{1}^{u}}{r_{2}^{l}} & =\frac{10}{9} \approx 1.11, \\
M_{2} & =\frac{1}{a_{2}^{l}} \exp \left(r_{2}^{u}-1\right)=2.5, \\
\frac{a_{1}^{l} e_{1}^{u}+c_{1}^{l} d_{1}^{l}}{b_{2}^{u} e_{1}^{u}} & \approx 3.11, \\
\frac{b_{1}^{l} e_{2}^{l}}{1+M_{2}} \frac{a_{2}^{u} e_{2}^{l}+c_{2}^{u} d_{2}^{u}}{A_{22}} & =\min \left\{a_{2}^{l}, \frac{2}{M_{2}}-a_{2}^{u}\right\}=0.4, \\
0.1 & =c_{2}^{u}<\frac{A_{22} e_{2}^{l}}{d_{2}^{u}}=0.12 .
\end{aligned}
$$

Clearly, condition $\left(H_{1}\right)$ and (68) are satisfied, and so from Theorem 2 and 4 we know that species $x_{1}$ will be driven to extinction, while species $x_{2}$ is global attractive.

Figure 2 shows the dynamic behaviors of system (86), which strongly support our results.

Example 3. Consider the following equations:

$$
\begin{aligned}
& x_{1}(k+1)=x_{1}(k) \exp \left\{1-(2.5+0.5 \sin (k)) x_{1}(k)-\frac{5 \sum_{s=0}^{\infty}((e-1) / e) e^{-s} x_{2}(k-s)}{1+\sum_{s=0}^{\infty}((e-1) / e) e^{-s} x_{2}(k-s)}-0.00005 x(k) x_{2}^{2}(k)-0.1 \sum_{s=0}^{\infty} \frac{e^{2}-1}{e^{2}} e^{-2 s} u_{1}(k-s)\right\} \\
& x_{2}(k+1)=x_{2}(k) \exp \left\{0.65-0.05 \cos (k)-\frac{5 \sum_{s=0}^{\infty}((e-1) / e) e^{-s} x_{1}(k-s)}{1+\sum_{s=0}^{\infty}((e-1) / e) e^{-s} x_{1}(k-s)}-3 x_{2}(k)-0.3 \sum_{s=0}^{\infty} \frac{e^{2}-1}{e^{2}} e^{-2 s} u_{2}(k-s)\right\} \\
& u_{1}(k+1)=u_{1}(k)(1-0.9)+5 \sum_{s=0}^{\infty} \frac{e^{3}-1}{e^{3}} e^{-3 s} x_{1}(k-s), \\
& u_{2}(k+1)=u_{2}(k)(1-0.9)+2 \sum_{s=0}^{\infty} \frac{e^{3}-1}{e^{3}} e^{-3 s} x_{2}(k-s) .
\end{aligned}
$$



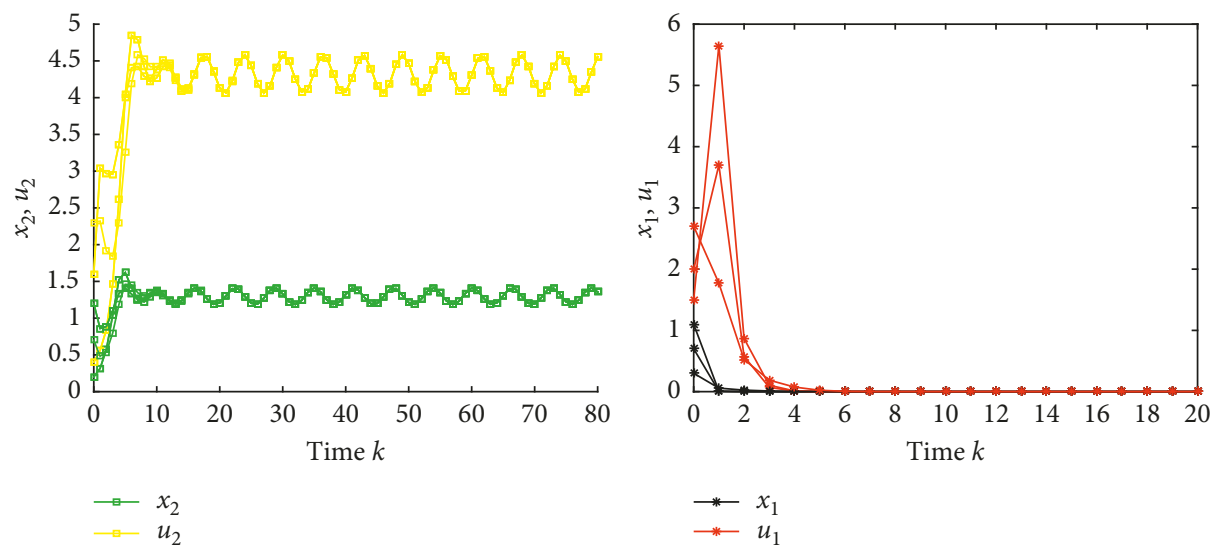

Figure 2: Numeric simulations of the solutions $\left(x_{1}(k), x_{2}(k), u_{1}(k), u_{2}(k)\right)$ of system (86), with the initial conditions $\left(x_{1}(s), x_{2}(s), u_{1}(s), u_{2}(s)\right)=(0.3,0.2,2.7,0.4),(0.7,1.2,2,1.6),(1.1,0.7,1.5,2.3), s=\ldots,-n,-n+1, \ldots,-1,0$, respectively.

One could easily see that conditions $\left(C_{1}\right)$ and $\left(C_{2}\right)$ are satisfied. Also, by calculating, one has

$$
\begin{aligned}
M_{1} & =\frac{1}{a_{1}^{l}} \exp \left(r_{1}^{u}-1\right)=0.5, \\
M_{2} & =\frac{1}{a_{2}^{l}} \exp \left(r_{2}^{u}-1\right) \approx 0.25, \\
\left(1+M_{1}\right) \frac{a_{1}^{u} e_{1}^{l}+c_{1}^{u} d_{1}^{u}}{b_{2}^{l} e_{1}^{l}} & \approx 1.07, \\
\frac{b_{1}^{u} e_{2}^{u}}{a_{2}^{l} e_{2}^{u}+c_{2}^{l} d_{2}^{l}} & \approx 1.36, \\
\frac{r_{1}^{l}}{r_{2}^{u}} & \approx 1.43>\max \{1.07,1.36\},
\end{aligned}
$$$$
\frac{1}{M_{1} M_{2}^{2}}\left(r_{1}^{l}-\left(1+M_{1}\right) r_{2}^{u} \frac{a_{1}^{u} e_{1}^{l}+c_{1}^{u} d_{1}^{u}}{b_{2}^{l} e_{1}^{l}}\right)=8
$$$$
\frac{1}{M_{1} M_{2}^{2}}\left(r_{1}^{l}-r_{2}^{u} \frac{b_{1}^{u} e_{2}^{u}}{a_{2}^{l} e_{2}^{u}+c_{2}^{l} d_{2}^{l}}\right)=1.6
$$$$
A_{11}=\min \left\{a_{1}^{l}, \frac{2}{M_{1}}-a_{1}^{u}\right\}=1,
$$$$
0.1=c_{1}^{u}<\frac{A_{11} e_{1}^{l}}{d_{1}^{u}}=0.18 .
$$

Clearly, conditions $\left(\mathrm{H}_{2}\right),\left(\mathrm{H}_{3}\right)$, and (70) are satisfied, and so from Theorem 3 and 5, we know that species $x_{2}$ will be driven to extinction, while species $x_{1}$ is global attractive.

Figure 3 shows the dynamics behaviors of system (88), which strongly supports our results.

\section{Discussion}

(1) From the conditions of Theorems 2-5, we can easily find that the feedback control variables and toxic substances play a crucial role on the extinction of system (5). We find that, by choosing suitable feedback control variable, one of the species will be extinct or permanent, that is, feedback control variable, which represents the biological control or some harvesting procedures, is an unstable factor of the system. From the conditions of Theorem 2, we also find that, despite the second species could produce toxicity, if the toxic rate is very low such that inequality $\left(\mathrm{H}_{3}\right)$ holds; then, the second species is still driven to extinction; in other words, that lower rate of toxic production has no influence on the extinction property of system (5).

(2) From the conditions of Theorem 1, we find that the feedback control variables and toxic substances play an important role on the permanence of the system; only the rate of toxic production and feedback control variables are small enough such that inequality (18) holds, and the toxic substances and feedback controls have no effect on the permanence of the system.

(3) $\mathrm{Yu}$ [7] obtained a set of sufficient conditions that guarantees the extinction of system (3), as direct corollaries of Theorems 2-5; one could also obtain other conditions for the extinction of system (3), which supplements and complements the results of $\mathrm{Yu}[7]$.

(4) Now, concerned with the extinction property of system (2), with some minor revisions to the proof of Theorems 2 and 3, we could obtain the following results.

Corollary 1. Assume that

$$
\frac{r_{1}^{u}}{r_{2}^{l}}<\min \left\{\frac{a_{1}^{l}}{b_{2}^{u}}, \frac{b_{1}^{l}}{\left(1+M_{2}\right) a_{2}^{u}}\right\}
$$

holds, and let $\left(x_{1}(k), x_{2}(k)\right)$ be any positive solution of system (2), then

$$
\lim _{k \longrightarrow+\infty} x_{1}(k)=0 .
$$



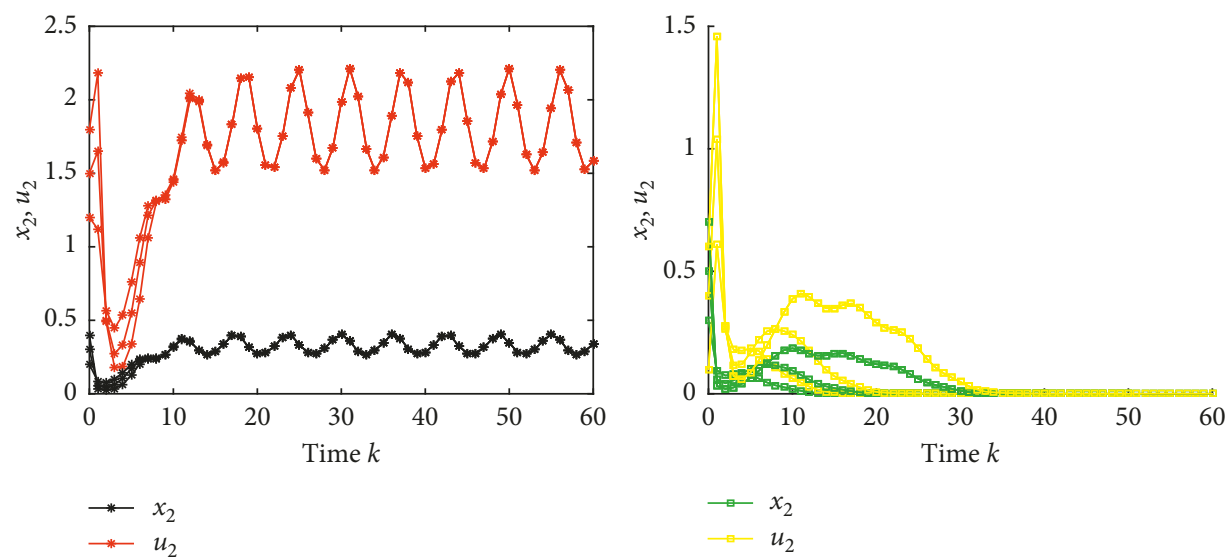

Figure 3: Numeric simulations of the solutions $\left(x_{1}(k), x_{2}(k), u_{1}(k), u_{2}(k)\right)$ of system (88), with the initial conditions $\left(x_{1}(s), x_{2}(s), u_{1}(s), u_{2}(s)\right)=(0.2,0.3,1.2,0.1),(0.3,0.5,1.5,0.4),(0.4,0.7,0.8,0.6), s=\ldots,-n,-n+1, \ldots,-1,0$, respectively.

Corollary 2. Assume that

$$
\begin{aligned}
& \frac{r_{1}^{l}}{r_{2}^{u}}>\max \left\{\left(1+M_{1}\right) \frac{a_{1}^{u}}{b_{2}^{l}}, \frac{b_{1}^{u}}{a_{2}^{l}}\right\}, \\
& \gamma^{u}<\min \frac{1}{M_{1} M_{2}^{2}}\left\{r_{1}^{l}-\left(1+M_{1}\right) r_{2}^{u} \frac{a_{1}^{u}}{b_{2}^{l}}, r_{1}^{l}-r_{2}^{u} \frac{b_{1}^{u}}{a_{2}^{l}}\right\},
\end{aligned}
$$

hold; let $\left(x_{1}(k), x_{2}(k)\right)$ be any positive solution of system (2), then

$$
\lim _{n \longrightarrow+\infty} x_{2}(k)=0 \text {. }
$$

It is interesting to investigate the stability property of the rest species under the assumption that one of the species in system (2) is driven to extinction. As direct corollaries of Theorems 4 and 5, by simple computation, we have

Corollary 3. Assume that (90) holds; also,

$$
\frac{a_{2}^{u}}{a_{2}^{l}} \exp \left\{r_{2}^{u}-1\right\}<2
$$

holds; then, for any positive solution $\left(x_{1}(k), x_{2}(k)\right)$ of system (2), we have

$$
\begin{aligned}
\lim _{n \longrightarrow+\infty} x_{1}(k) & =0, \\
\lim _{n \longrightarrow+\infty}\left(x_{2}(k)-x_{2}^{*}(k)\right) & =0,
\end{aligned}
$$

where $x_{2}^{*}(k)$ is any positive solution of the system $x_{2}(k+1)=x_{2}(k) \exp \left(r_{2}(k)-a_{2}(k) x_{2}(k)\right)$.

Corollary 4. Assume that (92) and (93) hold; also,

$$
\frac{a_{1}^{u}}{a_{1}^{l}} \exp \left\{r_{1}^{u}-1\right\}<2
$$

holds; then, for any positive solution $\left(x_{1}(k), x_{2}(k)\right)$ of system (2), we have

$$
\begin{aligned}
\lim _{n \longrightarrow+\infty} x_{2}(k) & =0, \\
\lim _{n \longrightarrow+\infty}\left(x_{1}(k)-x_{1}^{*}(k)\right) & =0,
\end{aligned}
$$

where $x_{1}^{*}(k)$ is any positive solution of the system $x_{1}(k+1)=x_{1}(k) \exp \left(r_{1}(k)-a_{1}(k) x_{1}(k)\right)$.

Obviously, Theorems 2.1, 2.5, 3.1, and 3.2 obtained by Yue [3] are corollaries 1-4 of Theorems 2-5, so corollaries 1-4 extend the results of Yue [3].

\section{Data Availability}

The data used to support the findings of this study are available from the corresponding author upon request.

\section{Conflicts of Interest}

The authors declare that there are no conflicts of interest.

\section{Authors' Contributions}

All authors contributed equally to the writing of this paper. All authors read and approved the final manuscript.

\section{Acknowledgments}

The research was supported by the Natural Science Foundations of China (no. 11771082) and the Scientific Research Development Fund of Young Researchers of Guangxi University of Finance and Economics (no. 2019QNB09).

\section{References}

[1] W. J. Qin, Z. J. Liu, and Y. P. Chen, "Permanence and global stability of positive periodic solutions of a discrete competitive system," Discrete Dynamics in Nature and Society, vol. 2009, Article ID 830537, 13 pages, 2009.

[2] Q. L. Wang and Z. J. Liu, "Uniformly asymptotic stability of positive almost periodic solutions for a discrete competitive system," Journal of Applied Mathematics, vol. 2013, Article ID 182158, 9 pages, 2013. 
[3] Q. Yue, "Extinction for a discrete competition system with the effect of toxic substances," Advances in Difference Equations, vol. 2016, no. 1, Article ID 1, 2016.

[4] X. D. Xie, Y. L. Xue, and R. X. Wu, "Global attractivity of a discrete competition model of plankton allelopathy with infinite deviating arguments," Advances in Difference Equations, vol. 2016, no. 1, Article ID 303, 2016.

[5] C. Xu and P. Li, "On the periodicity and global stability for a discrete delayed predator-prey model," International Journal of Mathematics, vol. 24, no. 10, Article ID 1350086, 2013.

[6] Q. Wang and Z. Liu, "Uniformly asymptotic stability of positive almost periodic solutions for a discrete competitive system," Journal of Applied Mathematics, vol. 2013, Article ID 182158, 9 pages, 2013.

[7] S. B. Yu, "Extinction for a discrete competition system with feedback controls," Advances in Difference Equations, vol. 2017, no. 1, Article ID 9, 2017.

[8] L. Zhao, X. Xie, L. Yang et al., "Dynamic behaviors of a discrete lotka-volterra competition system with infinite delays and single feedback control," Abstract and Applied Analysis, vol. 2014, Article ID 867313, 19 pages, 2014.

[9] J. H. Chen and X. D. Xie, "Stability analysis of a discrete competitive system with nonlinear interinhibition terms," Advances in Difference Equations, vol. 2017, no. 1, Article ID 294, 2017.

[10] Y. Chen and Z. Zhou, "Stable periodic solution of a discrete periodic Lotka-Volterra competition system," Journal of Mathematical Analysis and Applications, vol. 277, no. 1, pp. 358-366, 2003.

[11] J. Xu, Z. Teng, and H. Jiang, "Permanence and global attractivity for discrete nonautonomous two-species LotkaVolterra competitive system with delays and feedback controls," Periodica Mathematica Hungarica, vol. 63, no. 1, pp. 19-45, 2011.

[12] Y. Muroya, "Persistence and global stability for discrete models of nonautonomous Lotka-Volterra type," Journal of Mathematical Analysis and Applications, vol. 273, no. 2, pp. 492-511, 2002.

[13] Y. Muroya, "Persistence and global stability in discrete models of Lotka-Volterra type," Journal of Mathematical Analysis and Applications, vol. 330, no. 1, pp. 24-33, 2007.

[14] L. Zhang, Z. D. Teng, T. Zhang, and S. Gao, "Extinction in nonautonomous discrete Lotka-Volterra competitive system with pure delays and feedback controls," Discrete Dynamics in Nature and Society, vol. 2009, Article ID 656549, 19 pages, 2009.

[15] G. Chen and Z. Teng, "On the stability in a discrete twospecies competition system," Journal of Applied Mathematics and Computing, vol. 38, no. 1-2, pp. 25-39, 2012.

[16] X. Kong, L. Chen, and W. Yang, "Note on the persistent property of a discrete lotka-volterra competitive system with delays and feedback controls," Advances in Difference Equations, vol. 2010, pp. 1-9, 2010.

[17] D. Y. Wu, "Global attractivity of a discrete lotka-Volterra competition system with infinite delays and feedback controls," Advances in Difference Equations, vol. 2013, no. 1, Article ID 14, 2013.

[18] D. Y. Wu and H. Z., "Bifurcation analysis of a two-species competitive discrete model of plankton allelopathy," $A d$ vances in Difference Equations, vol. 2014, no. 1, Article ID 70, 2014.
[19] Q. Y. Li, H. W. Liu, and F. Q. Zhang, "The permanence and extinction of a discrete predator-prey system with time delay and feedback controls," Advances in Difference Equations, vol. 2010, Article ID 738306, 20 pages, 2010.

[20] S. Choo, "Global stability in $n$-dimensional discrete LotkaVolterra predator-prey models," Advances in Difference Equations, vol. 2014, no. 1, Article ID 11, 2014.

[21] Z. X. Yu and Z. Li, "Permanence and global attractivity of a discrete two-prey one-predator model with infinite delay," Discrete Dynamics in Nature and Society, vol. 2009, Article ID 732510, 16 pages, 2009.

[22] L. J. Chen, X. D. Xie, and L. J. Chen, "Feedback control variables have no influence on the permanence of a discrete N-Species cooperation system," Discrete Dynamics in Nature and Society, vol. 2009, Article ID 306425, 10 pages, 2009.

[23] Y. Xue, X. Xie, Q. Lin, and F. Chen, "Global attractivity and extinction of a discrete competitive system with infinite delays and single feedback control," Discrete Dynamics in Nature and Society, vol. 2018, Article ID 1893181, 14 pages, 2018.

[24] X. D. Xie, Y. L. Xue, R. Wu, and L. Zhao, "Extinction of a two species competitive system with nonlinear inter-inhibition terms and one toxin producing phytoplankton," Advances in Difference Equations, vol. 2016, no. 1, Article ID 258, 2016.

[25] J. Solé, E. García-Ladona, P. Ruardij, and M. Estrada, "Modelling allelopathy among marine algae," Ecological Modelling, vol. 183, no. 4, pp. 373-384, 2005.

[26] M. Bandyopadhyay, "Dynamical analysis of a allelopathic phytoplankton model," Journal of Biological Systems, vol. 14, no. 2, pp. 205-217, 2006.

[27] B. Chen, "Permanence for the discrete competition model with infinite deviating arguments," Discrete Dynamics in Nature and Society, vol. 2016, Article ID 1686973, 5 pages, 2016.

[28] C. J. Xu, Y. S. Wu, and L. Li, "Permanence and global attractivity in a discrete Lotka-Volterra predator-prey model with delays," Advances in Difference Equations, vol. 208, pp. 1-15, 2014.

[29] C. Xu and P. Li, "Oscillations for a delayed predator-prey model with Hassell-Varley-type functional response," Comptes Rendus Biologies, vol. 338, no. 4, pp. 227-240, 2015.

[30] C. Xu and Y. Zu, "Permanence of a two species delayed competitive model with stage structure and harvesting," Bulletin of the Korean Mathematical Society, vol. 52, no. 4, pp. 1069-1076, 2015.

[31] C. J. Xu, "Delay-induced oscillations in a competitor-competitor-mutualist lotka-volterra model," Complexity, vol. 2017, Article ID 2578043, 12 pages, 2017.

[32] C. Xu, P. Li, and Y. Guo, "Global asymptotical stability of almost periodic solutions for a non-autonomous competing model with time-varying delays and feedback controls," Journal of Biological Dynamics, vol. 13, no. 1, pp. 407-421, 2019.

[33] C. Xu and M. Liao, "Bifurcation analysis of an autonomous epidemic predator-prey model with delay," Annali di Matematica Pura ed Applicata, vol. 193, no. 1, pp. 23-38, 2014.

[34] X. Xie, Y. Xue, and R. Wu, "Global attractivity in a discrete mutualism model with infinite deviating arguments," Discrete Dynamics in Nature and Society, vol. 2017, Article ID 2912147, 8 pages, 2017.

[35] S. P. Lu and X. C. Yu, "Existence of positive periodic solutions for Linard equations with an indefinite singularity of 
attractive type," Boundary Value Problems, vol. 2018, no. 1, p. 101, 2018.

[36] V. Slyn'ko and C. Tunç, "Stability of abstract linear switched impulsive differential equations," Automatica, vol. 107, pp. 433-441, 2019.

[37] L. Wang and M. Q. Wang, Ordinary Difference Equation, Xinjiang University Press, China, 1991, in Chinese.

[38] F. Chen, "Permanence in a discrete Lotka-Volterra competition model with deviating arguments," Nonlinear Analysis: Real World Applications, vol. 9, no. 5, pp. 2150-2155, 2008. 


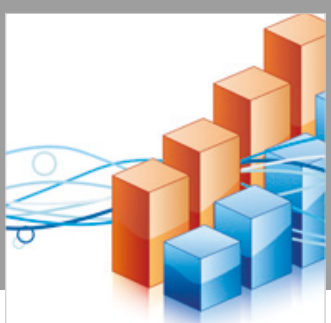

Advances in

Operations Research

\section{-n-m}
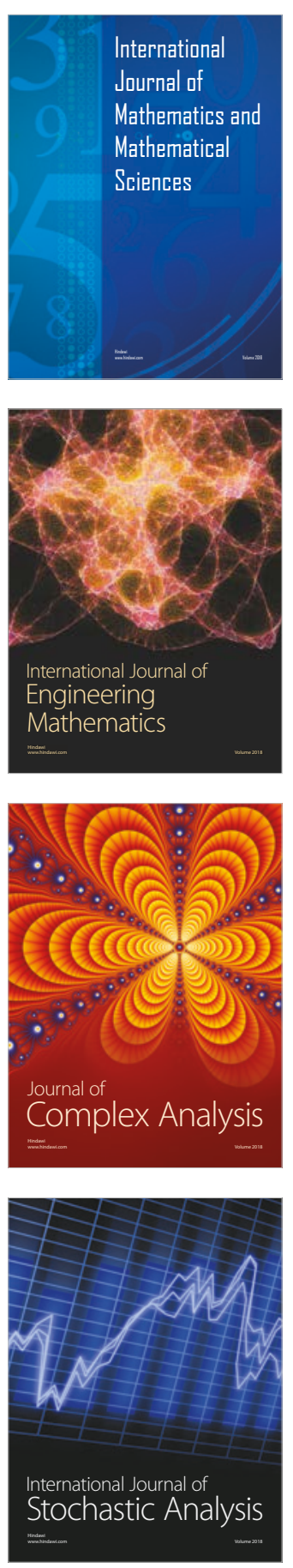
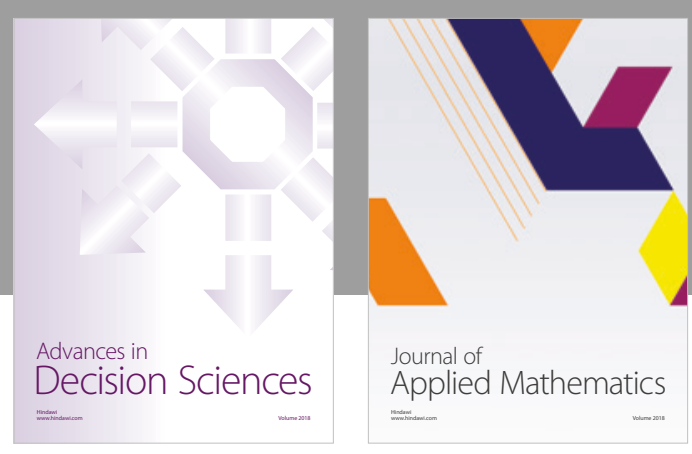

Journal of

Applied Mathematics
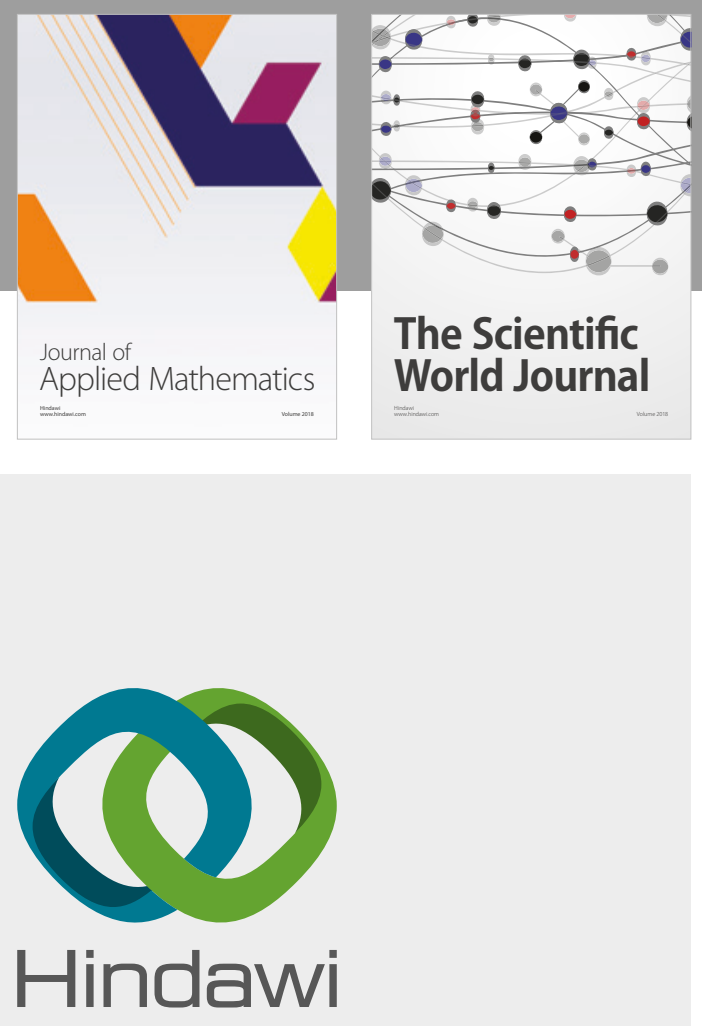

Submit your manuscripts at

www.hindawi.com

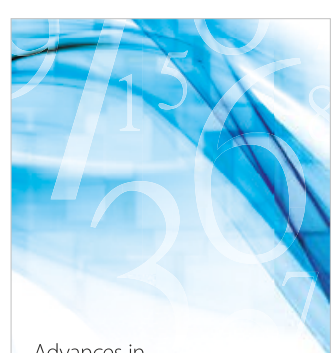

Advances in
Numerical Analysis
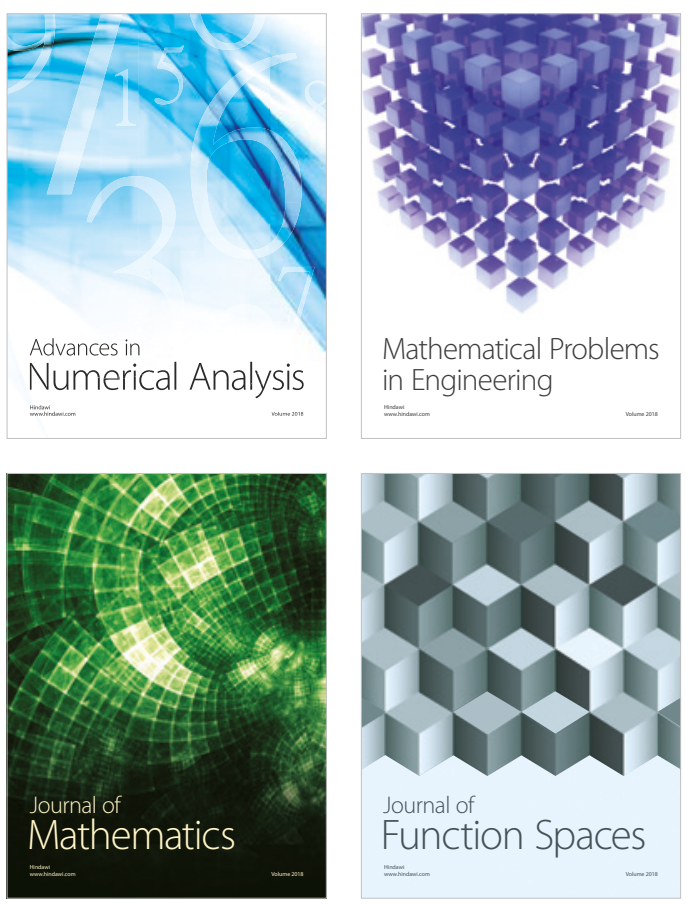

Mathematical Problems in Engineering

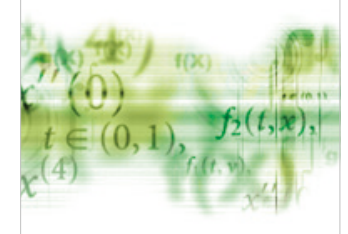

International Journal of

Differential Equations

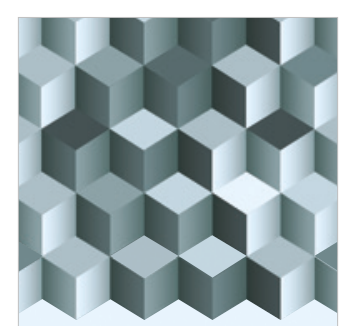

Journal of

Function Spaces
The Scientific

World Journal

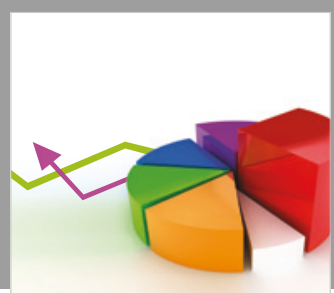

Journal of

Probability and Statistics
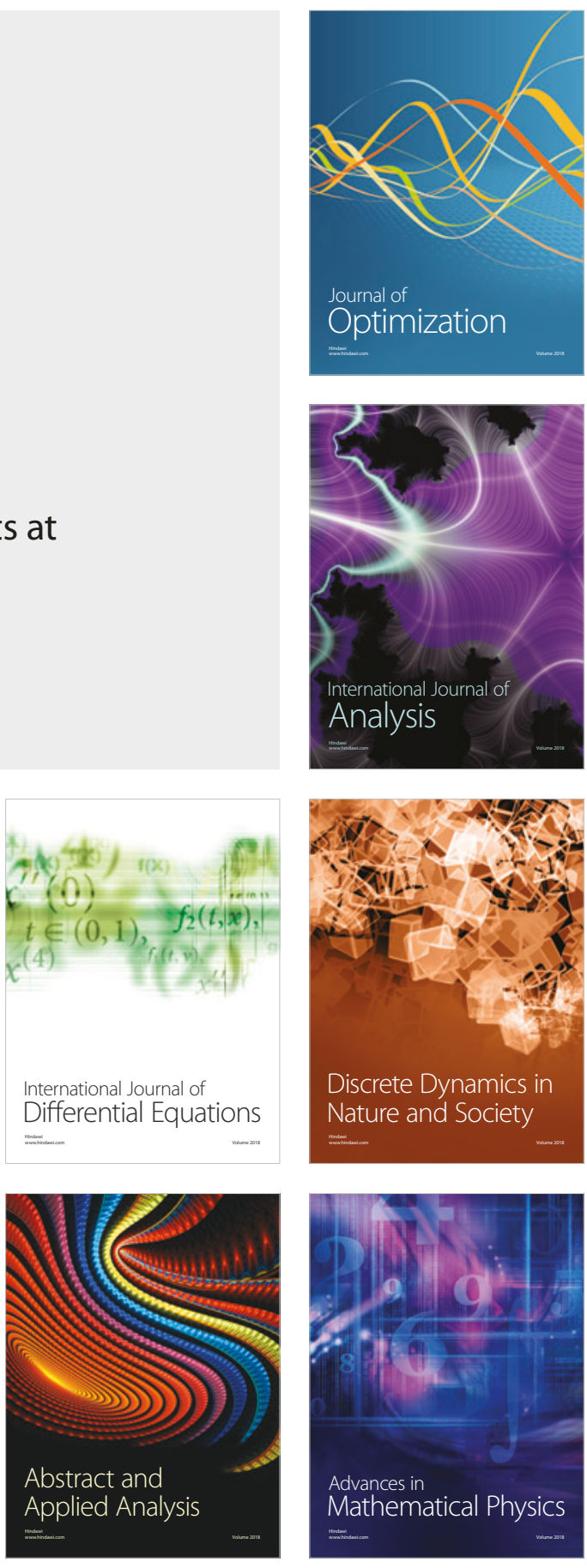